Article

\title{
Landslide Displacement Prediction Method Based on GA-Elman Model
}

\author{
Chenhui Wang ${ }^{1, t, \ddagger(\mathbb{D}}$, Yijiu Zhao ${ }^{1, \ddagger \mathbb{D}}$, Libing Bai ${ }^{1, \ddagger}$, Wei Guo ${ }^{2, t, \ddagger}$ and Qingjia Meng ${ }^{2, *, t, \ddagger(\mathbb{D}}$ \\ 1 School of Automation Engineering, University of Electronic Science and Technology of China; \\ Chengdu 611731, China; wangchenhui@mail.cgs.gov.cn (C.W.); yijiuzhao@uestc.edu.cn (Y.Z.); \\ libing.bai@uestc.edu.cn (L.B.) \\ 2 Technology Innovation Center for Geological Environment Monitoring, Ministry of Natural Resources, \\ Baoding 071051, China; gwei_b@mail.cgs.gov.cn \\ * Correspondence: mengqingjia@mail.cgs.gov.cn; Tel.: +86-0312-5908723 \\ + Current address: Center for Hydrogeology and Environmental Geology Survey, China Geology Survey, \\ Baoding 071051, China. \\ $\ddagger \quad$ These authors contributed equally to this work.
}

Citation: Wang, C.; Zhao Y.; Bai L.; Guo, W.; Meng, Q. Landslide Displacement Prediction Method Based on GA-Elman Model. Appl. Sci. 2021, 11, 11030. https://doi.org/ 10.3390/app112211030

Academic Editor: José A. F. O. Correia

Received: 1 November 2021

Accepted: 15 November 2021

Published: 21 November 2021

Publisher's Note: MDPI stays neutral with regard to jurisdictional claims in published maps and institutional affiliations.

Copyright: (c) 2021 by the authors. Licensee MDPI, Basel, Switzerland. This article is an open access article distributed under the terms and conditions of the Creative Commons Attribution (CC BY) license (https:// creativecommons.org/licenses/by/ $4.0 /)$.
Abstract: The deformation process of landslide displacement has complex nonlinear characteristics. In view of the problems of large error, slow convergence and poor stability of the traditional neural network prediction model, in order to better realize the accurate and effective prediction of landslide displacement, this research proposes a landslide displacement prediction model based on Genetic Algorithm (GA) optimized Elman neural network. This model combines the GA with the Elman neural network to optimize the weights, thresholds and the number of hidden neurons of the Elman neural network. It gives full play to the dynamic memory function of the Elman neural network, overcomes the problems that a single Elman neural network can easily fall into local minimums and the neuron data is difficult to determine, thereby effectively improving the prediction performance of the neural network prediction model. The displacement monitoring data of a slow-varying landslide in the Guizhou karst mountainous area are selected to predict and verify the landslide displacement, and the results are compared with the traditional Elman neural network prediction results. The results show that the prediction results of GA-Elman model are in good agreement with the actual monitoring data of landslide. The average error of the model is low and the prediction accuracy is high, which proves that the GA-Elman model can play a role in the prediction of landslide displacement and can provide reference for the early warning of landslide displacement deformation.

Keywords: landslide displacement; prediction model; genetic algorithm; Elman neural network

\section{Introduction}

Landslide is one of the most serious geological hazards in the world $[1,2]$. The formation mechanism of landslide disaster is complex and diverse. Limited by different geological environment conditions, many influencing factors will lead to the occurrence of landslide [1]. Prediction of landslide deformation and deformation evolution is a difficult and crucial problem [3]. The evolution process of landslide is a complex nonlinear process of superposition of multiple influencing factors, so the long-term displacement monitoring data of landslide can be used to vividly represent the nonlinear displacement dynamic behavior characteristics. Therefore, landslide displacement prediction is an important part of landslide disaster monitoring and early warning system $[4,5]$. Determining how to design an effective landslide deformation monitoring and prediction method is helpful to understand the instability process and deformation characteristics of landslide disasters, which is conducive to reducing the risks of landslide to human life and property safety and infrastructure [6].

The main characteristics of landslide deformation are the change of crack displacement on the landslide body and the deformation of displacement in the key deformation 
area. Therefore, it is particularly important to carry out deformation monitoring on the relative displacement and absolute displacement changes of landslide. Timely prediction of landslide displacement and deformation has important reference value for judging landslide deformation trend. Researchers have carried out a number of studies on landslide displacement prediction and have made some progress. For example, the empirical model is used to simulate the landslide displacement monitoring data. The common empirical models include Saito model, Hayashi model and Voight model. The above model uses the theoretical model of landslide creep to predict the displacement and deformation of landslide through a large number of experimental data. However, when the landslide is affected by other external factors, the model parameters need to be adjusted in time, otherwise the accuracy of the model will be reduced and the prediction effect will be affected. In addition, researchers also designed a series of landslide displacement prediction models through mathematical statistics, including grey system model [7], plant growth model and grey displacement vector angle model [8]. For example, Miao et al. (2017) used grey model to construct different types of landslide displacement prediction methods [3].

With the continuous updating of artificial intelligence algorithms, some nonlinear models have been applied to landslide displacement prediction. The traditional nonlinear models mainly include catastrophe theory [9], cooperative work theory [10], back propagation neural network model [11,12], support vector regression model $[4,13,14]$, chaos model [15-17], decision tree model [18,19], extreme learning machine model [20,21], Elman neural network model $[11,14,22-24]$ and so on. In the above models, Elman neural network has been widely applied to solve nonlinear system problems. The biggest difference between Elman neural network and traditional Back Propagation (BP) is that it has local memory function. Elman neural network is suitable for the prediction of time series because of its parallel processing, self-adaptation, self-learning and strong robustness. At present, some research scholars have carried out relevant research on the predictive evaluation of Elman neural network, such as Bai et al. (2012), who used the Elman neural network model to verify the feasibility of monitoring and forecasting landslides [25]. According to the nonlinear characteristics of landslide displacement monitoring data, Chen et al. (2013) proposed an improved Elman recurrent neural network and proved the accuracy of Elman neural network in short-term prediction [26]. Li et al. (2018) realized the dynamic prediction of loess landslide monitoring data through the Elman neural network and got a better prediction effect [27]. Li et al. (2015) constructed the rolling prediction model of open-pit slope by combining local mean decomposition with Elman neural network [28]. However, Elman neural network also has some common shortcomings of neural network. It mainly includes the disadvantages of self-existence weight updating method, which are that it easily falls into local minimum solution, hidden neurons need to be determined by trial and error repeatedly and slow convergence rate.

To solve these problems, a landslide displacement prediction method based on adaptive genetic algorithm and Elman neural network model is proposed in this study. The displacement monitoring data of a slow-varying landslide in Guizhou karst mountainous area are selected to predict and verify the landslide displacement. This study analyzes the internal development process and external environmental factors of the landslide. The weight, threshold and network structure of Elman neural network are optimized by using adaptive genetic algorithm (GA), which can effectively solve the problem that it easily falls into local minimum solution. This model can improve the prediction performance of the whole neural network and effectively improve the accuracy of landslide displacement prediction.

\section{Method}

2.1. GA-Elman Displacement Prediction Model

2.1.1. Elman Neural Network

Elman neural network is a feedback recurrent neural network with local memory function [24]. The least square method is used to realize the minimum mean square 
deviation of the actual output and the expected output of the neural network, which can better reflect the dynamics of the system. The Elman neural network also has the input layer, hidden layer and output layer similar to the neural network, and also has a special structure layer: the context layer (state layer). The significance of the context layer is to memorize the output value of the hidden layer at the previous moment, which can be considered as a one-step delay operator and enhance the internal feedback mechanism of the network. It can make the neural network have better nonlinear and time-varying characteristics, and effectively improve the global stability of the neural network. The learning process of the neural network consists of two parts: the forward propagation of the signal and the back propagation of the error. Through the repeated training of these two parts, the weight value and threshold value between the input layer and the hidden layer and between the hidden layer and the output layer can be continuously developed to the direction of error reduction, making the prediction results more close to the measured results [26]. The structure of Elman neural network is shown in Figure 1.

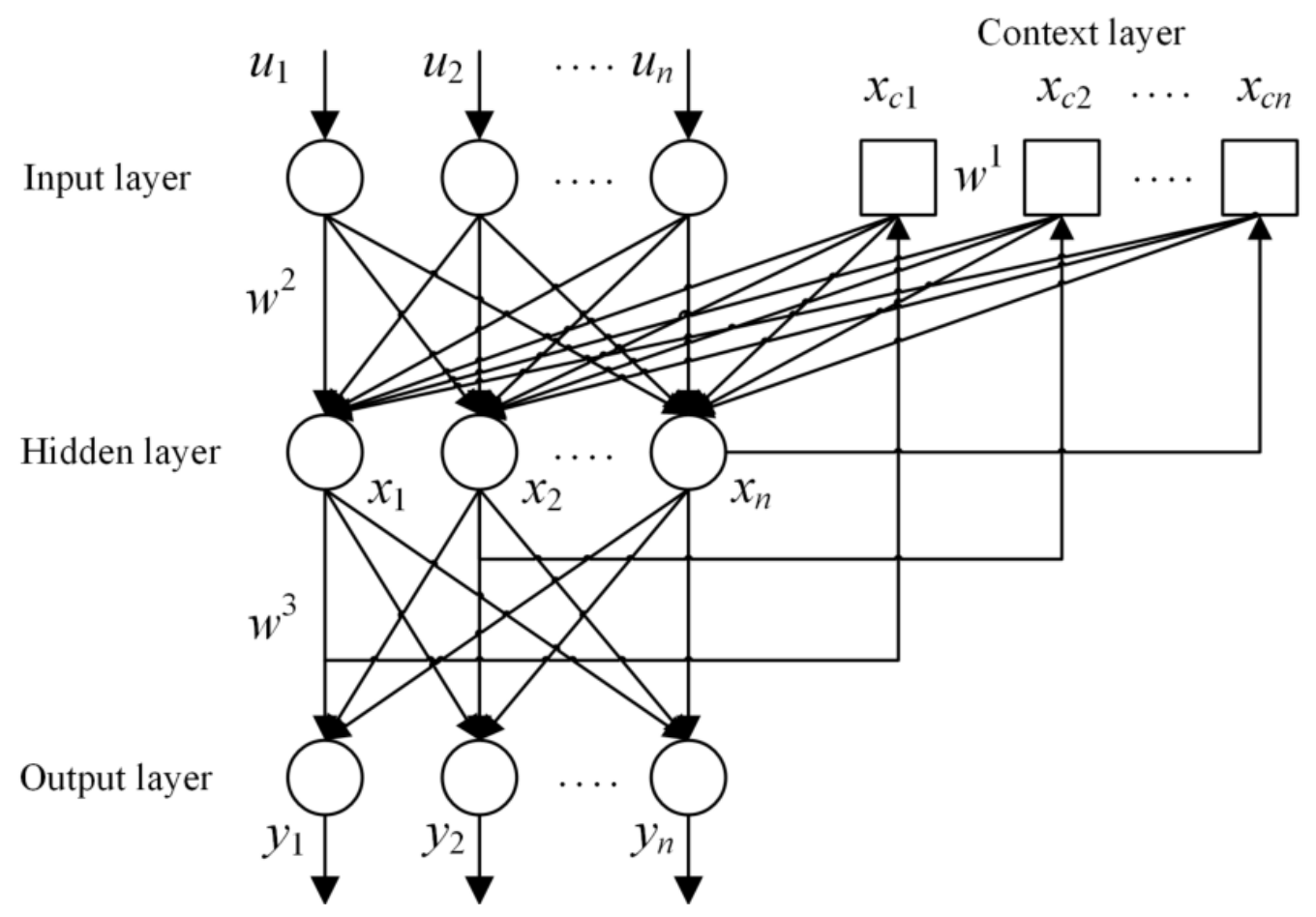

Figure 1. Structure of Elman neural network.

The formation mechanism of landslide disaster is complex and there are many factors leading to landslide deformation, including rainfall infiltration, groundwater activity, earthquake, human engineering excavation and so on. Therefore, these factors should be fully considered in the design of input layers for reasonable selection and the neural network model should be optimized to improve the practicability of the model. According to the neural network model, the mathematical model of Elman neural network can be expressed as:

$$
\left\{\begin{array}{l}
y(k)=g\left(w^{3} x(k)\right) \\
x(k)=f\left(w^{1} x_{c}(k)+w^{2}(u(k-1))\right) \\
x_{c}(k)=x(k-1)
\end{array}\right.
$$

$u(k)=\left[u_{1}(k), u_{2}(k), \cdots, u_{n}(k)\right]$ is the input vector. The function of the input vector is to transmit data to the neural network.

$y(k)=\left[y_{1}(k), y_{2}(k), \cdots, y_{n}(k)\right]$ is the output vector. The output vector plays a linear weighting role in the network structure.

$x(k)=\left[x_{1}(k), x_{2}(k), \cdots, x_{n}(k)\right]$ is the output vector of hidden layer. The hidden layer is used as a transfer function. 
$x_{c}(k)=\left[x_{c 1}(k), x_{c 2}(k), \cdots, x_{c n}(k)\right]$ is the output layer of context layer. The context layer is used to memorize the output value of the previous time of the hidden layer and return the input to the network.

$w^{1}, w^{2}, w^{3}$ are weight matrices from context layer to hidden layer, input layer to hidden layer and hidden layer to output layer, respectively.

In this study, "tansig" nonlinear function is used as the transfer function of the hidden layer and the transfer function $f(\cdot)$ of the hidden layer is a nonlinear function. In addition, the maximum training times and accuracy of the array will directly affect the reliability and accuracy of the neural network. When designing the number of hidden layers, the running time and accuracy of the neural network model should be considered. Due to the context layer, the Elman neural network has the so-called dynamic memory function. Its function is to memorize the output value of the hidden layer unit at the previous moment and return it to the input vector. In this paper, the transfer function $g(\cdot)$ of the output layer adopts the S-type logarithmic "logsig" function, which mainly plays a role of linear weighting.

\subsubsection{Construction of GA-Elman Model}

Genetic Algorithm (GA) is a global search algorithm with high parallelism, randomness and adaptability. The global search ability of genetic algorithm is usually used to solve the problems of multi-objective optimization, optimal route and feature selection. GA is widely used in the fields of training connection weights, designing network structure and finding optimal learning rules [29,30], the first two applications have been extensively studied by many scholars and have achieved good results. At present, most of the current research scholars are focusing on forward neural networks instead of the recurrent neural network[31,32]. Therefore, this study uses GA to optimize Elman neural network to solve the problem of neural network algorithm trapping in the local optimum and focuses on optimizing the initial weight and threshold of the Elman network.

\subsubsection{Workflow of GA-Elman Model}

The workflow of the GA-Elman neural network model is mainly as follows (Figure 2):

(1) Determine the topology of Elman neural network, mainly including the number of hidden layer neurons and other network topology parameters.

(2) Elman neural network initialization and initial weights and thresholds. GA encodes the initial weights and thresholds of the Elman network into an initial population composed of several chromosomes.

(3) Selection of fitness function. The key optimization goal of this research is the error between the output value of the neural network and the expected value, and the reciprocal of the sum of the squares of the actual output value and the expected value error is taken as the fitness function. The fitness function is calculated as shown in Equation (2).

$$
f(i)=\frac{1}{\sum_{i=1}^{n}\left(\hat{y}_{i}-y_{i}\right)^{2}}
$$

where $n$ is the number of network output nodes, $\hat{y}_{i}$ is the actual output value of the ith node of the Elman neural network and $y_{i}$ is the expected value of the ith node.

(4) Perform selection, crossover and mutation operations. The main purpose is to calculate the best fitness individual and eliminate the worst fitness individual. After repeated iterative optimization, the best neuron weights and thresholds of Elman neural network are obtained.

(5) The optimal weights and thresholds optimized by GA are passed to the Elman neural network. Elman network is trained and updated to calculate the error observation of neural network.

(6) Check whether the constraints are met and use the test set to verify the prediction effect. 


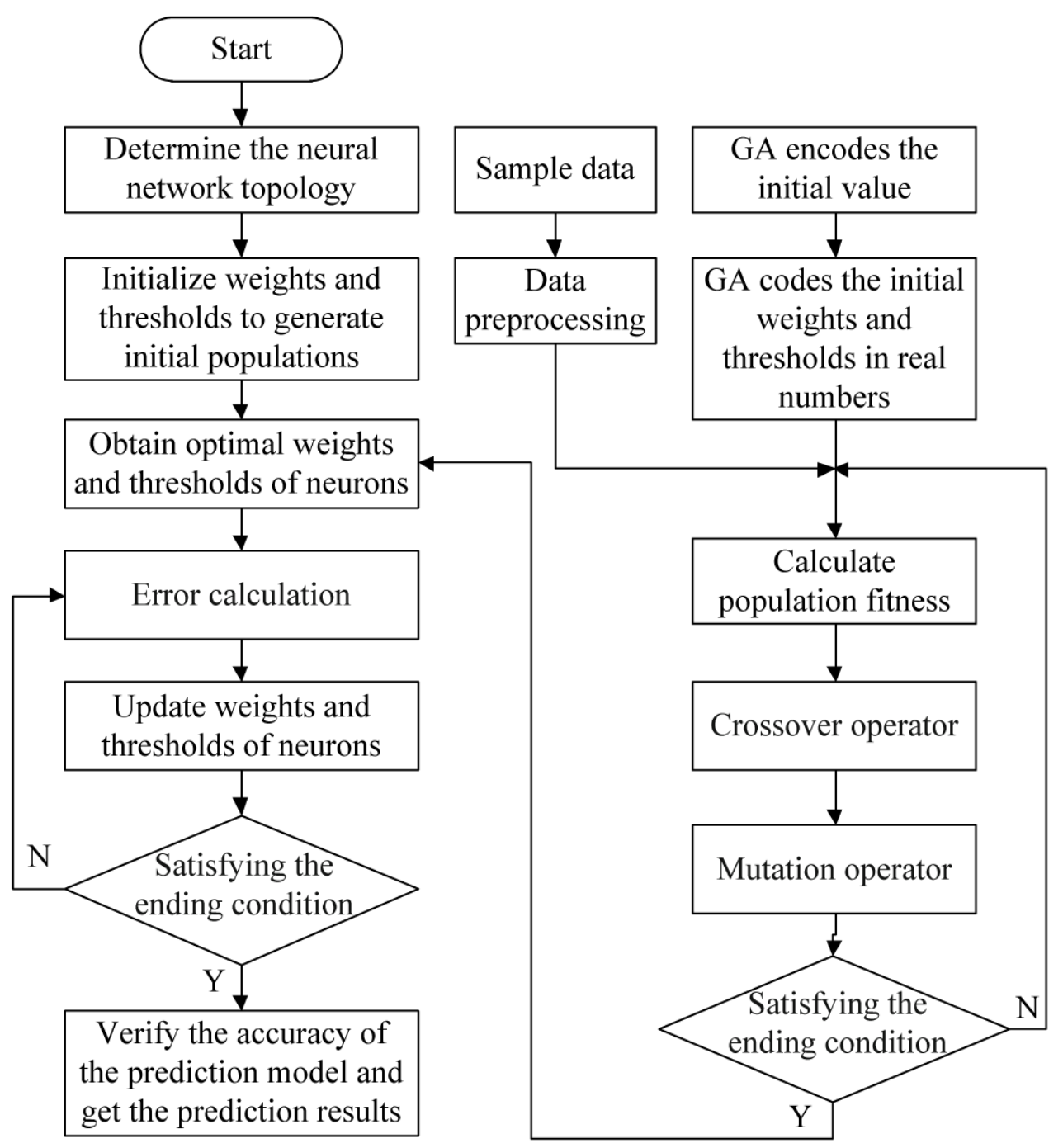

Figure 2. Flowchart of GA-Elman model.

\subsection{Performance Evaluation of Displacement Prediction Model}

In order to analyze the prediction ability of displacement monitoring data model, the evaluation index is used to analyze the displacement prediction performance, including root mean square error (RMSE), the mean absolute error (MAE), mean absolute percentage error (MAPE) and coefficient of determination $\left(R^{2}\right)$.

\section{Application Research and Method Comparison}

\subsection{Overview of the Study Area}

The Jianshanying landslide is located in the northwest of Faer Town, Shuicheng County, Liupanshui City, Guizhou Province, China. It is located in the western part of the Guizhou Plateau. The topography is up and down with strong cutting, and it belongs to the low-medium-low mountain landform formed by structural erosion. The landslide is high in the southeast and low in the northwest. The landslide boundary presents an irregular semicircle shape on the plane, including Jianshanying steep cliff and its lower gentle platform area. The front elevation range of the unstable slope deformation area is $1040-1120 \mathrm{~m}$ and the rear elevation range is $1380-1502 \mathrm{~m}$, with a height difference of about $483 \mathrm{~m}$. The rear wall of the slope is nearly steep and the front slope is about $63^{\circ}$. A total of five sets of monitoring equipment were deployed in the landslide, numbered EI19001-EI19005, respectively. The LoRa + 4 G method was used to collect the field crack displacement sensor data. As the gateway node, EI19001 is responsible for monitoring the rainfall in the landslide area. It is also responsible for obtaining the crack sensor monitoring 
data of other terminal nodes on the site. EI19002 EI19005 are the terminal nodes responsible for monitoring the changes of four key surface cracks in the landslide area. The schematic diagram of monitoring equipment deployment is shown in Figure 3.

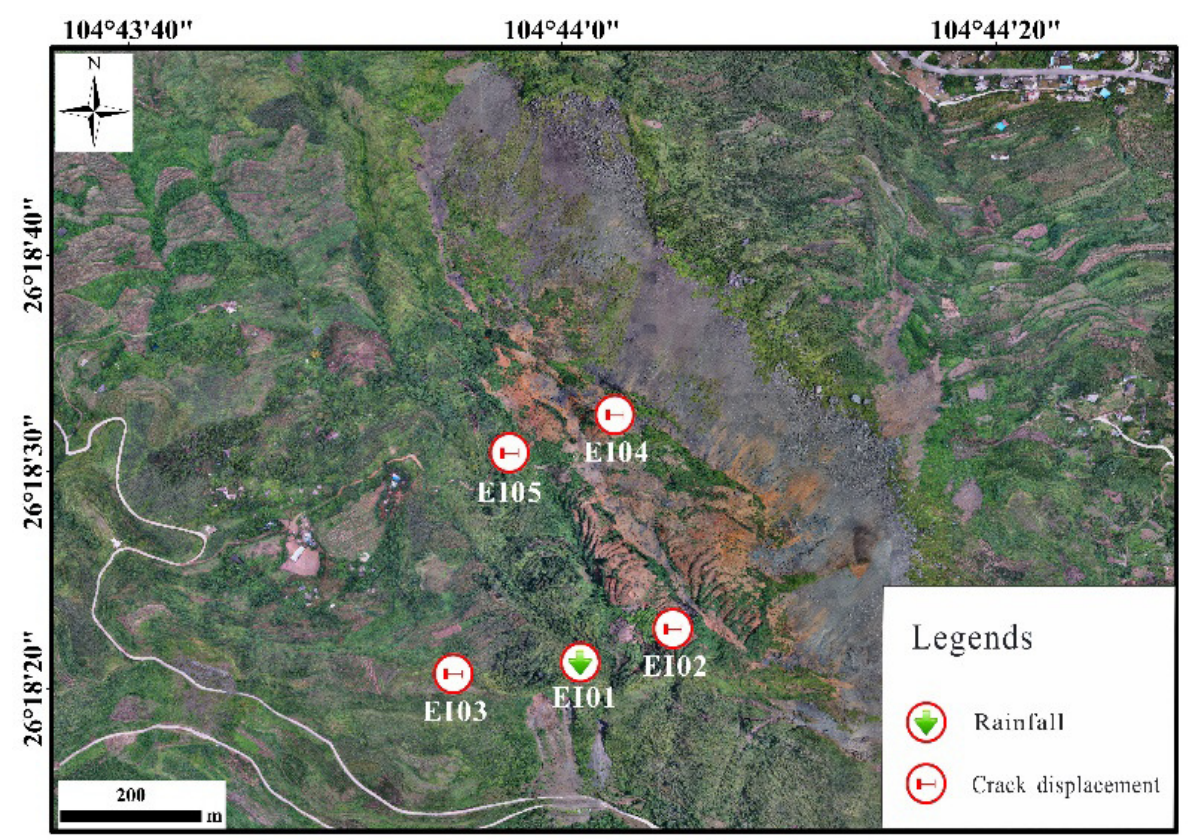

Figure 3. Schematic diagram of monitoring equipment deployment.

\subsection{Landslide Monitoring Data}

Four crack displacement monitoring points are set in the deformation area of the main cracks of the landslide, and the landslide monitoring data are accurately and reliably obtained by LoRa wireless ad hoc network communication. The low power frequency conversion mode and acquisition trigger mode are set, respectively. When the low power frequency conversion mode is used, the main frequency of the microprocessor is reduced from $16 \mathrm{MHz}$ to $65 \mathrm{KHz}$ and the device function module uploads the monitoring data regularly. When the trigger mode is collected, the microprocessor opens the real-time monitoring mode to monitor the change of cracks in real time. Once the preset threshold is exceeded, the data communication interface can start to upload the displacement monitoring data.

In this study, the monitoring data from 6 July 2020 to 30 October 2020 were selected for data analysis. The initial working mode of the system is set as periodic return interval of $1 \mathrm{~h}$, data collection port trigger return mode is enabled, rainfall trigger return threshold is set as $0.2 \mathrm{~mm}$ and displacement trigger return threshold is set as $20 \mathrm{~mm}$. Affected by rainfall factors, EI19003 began to change its displacement from 6 July 2020. The analysis of rainfall and crack displacement is shown in Figure 4. In order to explore the response relationship between rainfall and landslide crack displacement change, the displacement change during the rainfall cycle is used as the data research sample. The training set and the test set are constructed to predict the displacement change under the condition of rainfall and without rainfall, respectively. 


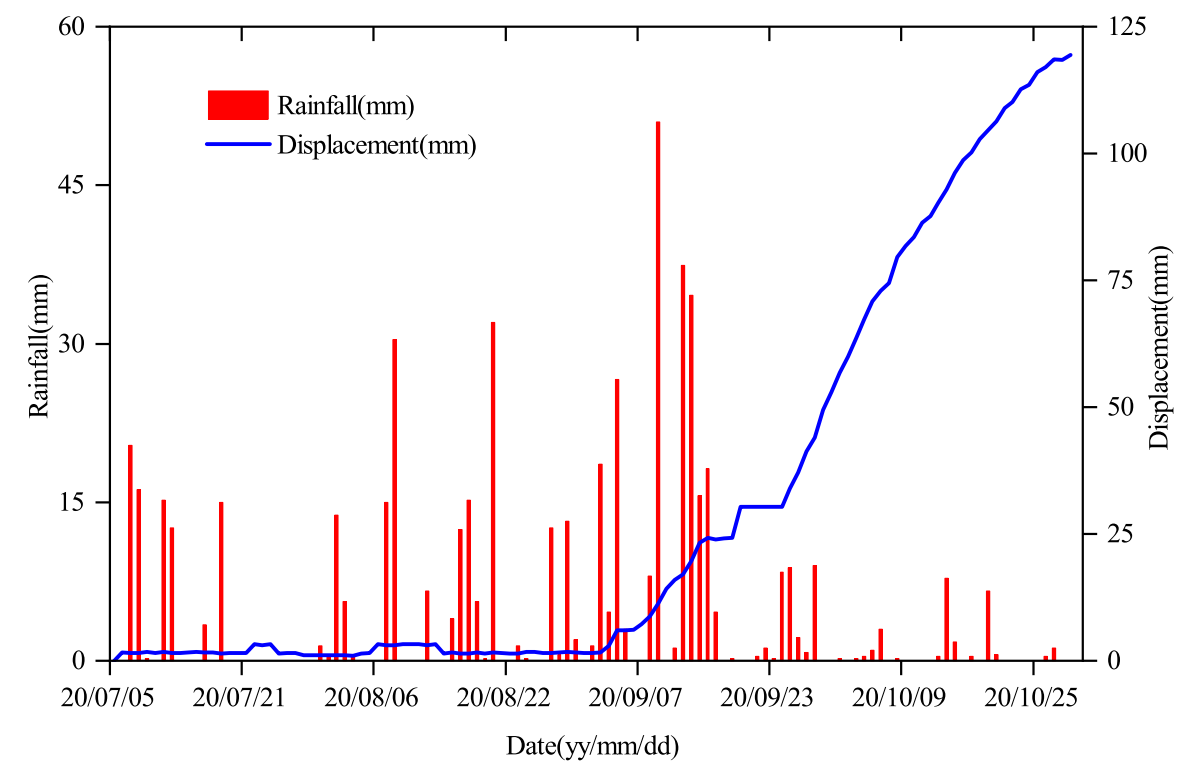

Figure 4. Comprehensive analysis curve of rainfall and displacement.

\subsection{Landslide Displacement Prediction of GA-Elman Model}

The weights and thresholds of Elman neural network are usually initialized by random numbers between intervals $[-0.5,0.5]$, and the initialization parameters have great influence on network training. Therefore, the purpose of this paper is to optimize the initial weight and threshold value of Elman neural network by GA[32,33]. The parameters that often need to be set in GA include population size, crossover rate, mutation rate, code length, etc. [34]. The setting of these parameters largely determines the operating performance of GA. According to the research result, the greater the crossover rate, the greater the chance of excellent individuals appearing, and the replacement of new and old individuals is quicker [35]. The empirical value is between $[0.25,1]$. The variation rate is generally between $[0,1]$.

In the network structure designed in this paper, the genetic algorithm runs for 50 generations, and the other control parameters are set as follows: population size: 40 , crossover rate: 0.7 , mutation rate: 0.01 , individual binary code length: 10 . According to the GA-Elman workflow introduced in 2.2, the first 97 days between 6 July 2020 and 30 October 2020 are selected as the training set, and the last 20 days of monitoring data are selected as the test set. Considering the rain factor and the non-rainfall factor respectively, the rolling forecast method is used to predict the displacement deformation trends for 1 day, 10 days and 20 days, respectively.

Figure 5 is the displacement time series of GA-Elman model for displacement training set data and prediction data. The results show that the effective and reasonable control parameters of GA provide a scientific and reliable input vector for the Elman model, and the displacement change can be effectively predicted by the constructed GA-Elman model.

After the GA-Elman model was used for training, a test set was selected to verify and analyze the trained model. Quantitative comparison was made between predicted values and test set. Figure 6 shows the prediction and comparison of GA-Elman model on test set.

Figure 6 shows that when the GA-Elman model continuously predicts 1 day, 10 days and 20 days, the predicted value of landslide displacement is very close to the actual value. The results show that a reasonable and reliable input vector is set for the construction of the GA-Elman model and the GA-Elman machine learning model can be used to accurately predict the landslide displacement. Figure 7 is the prediction and comparison of measured displacement and predicted displacement by GA-Elman model. The model has a certain convergence speed and accuracy, and has a relatively good adaptability, which can effectively and accurately predict the displacement change in advance. 


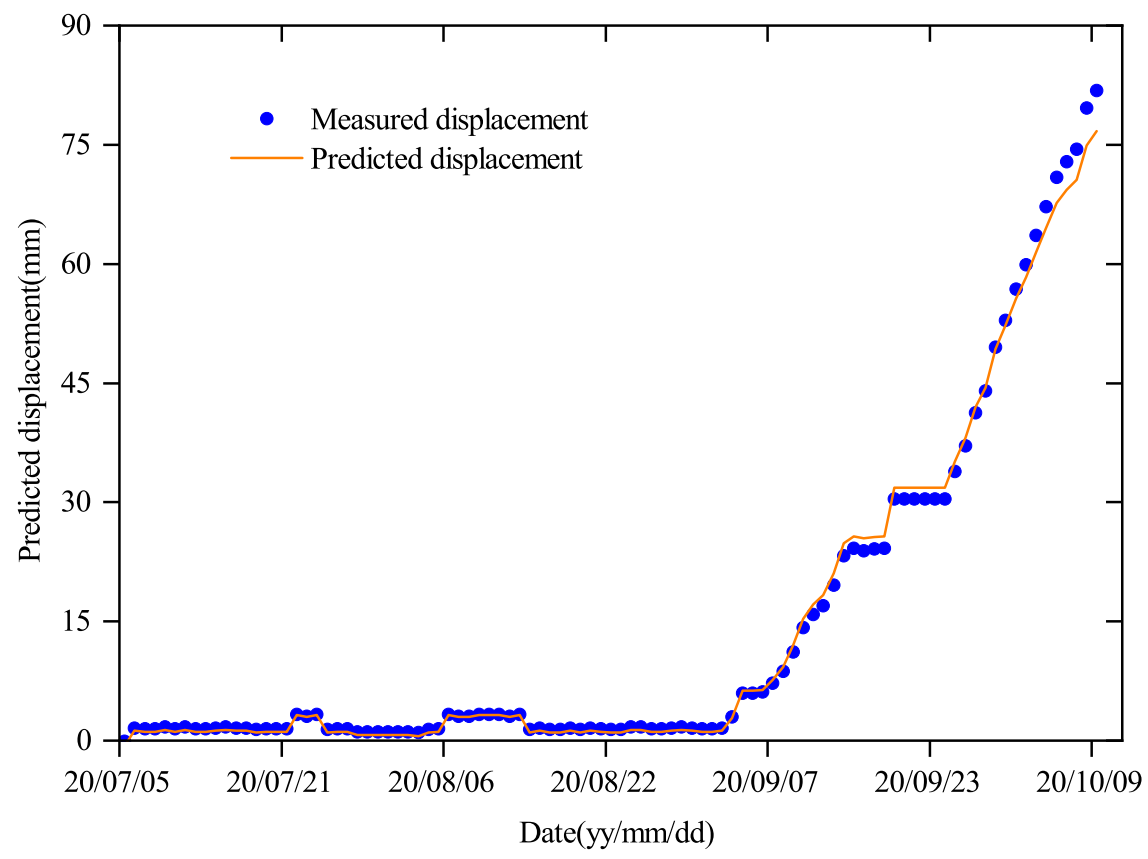

Figure 5. Prediction and comparison of GA-Elman model on training set.

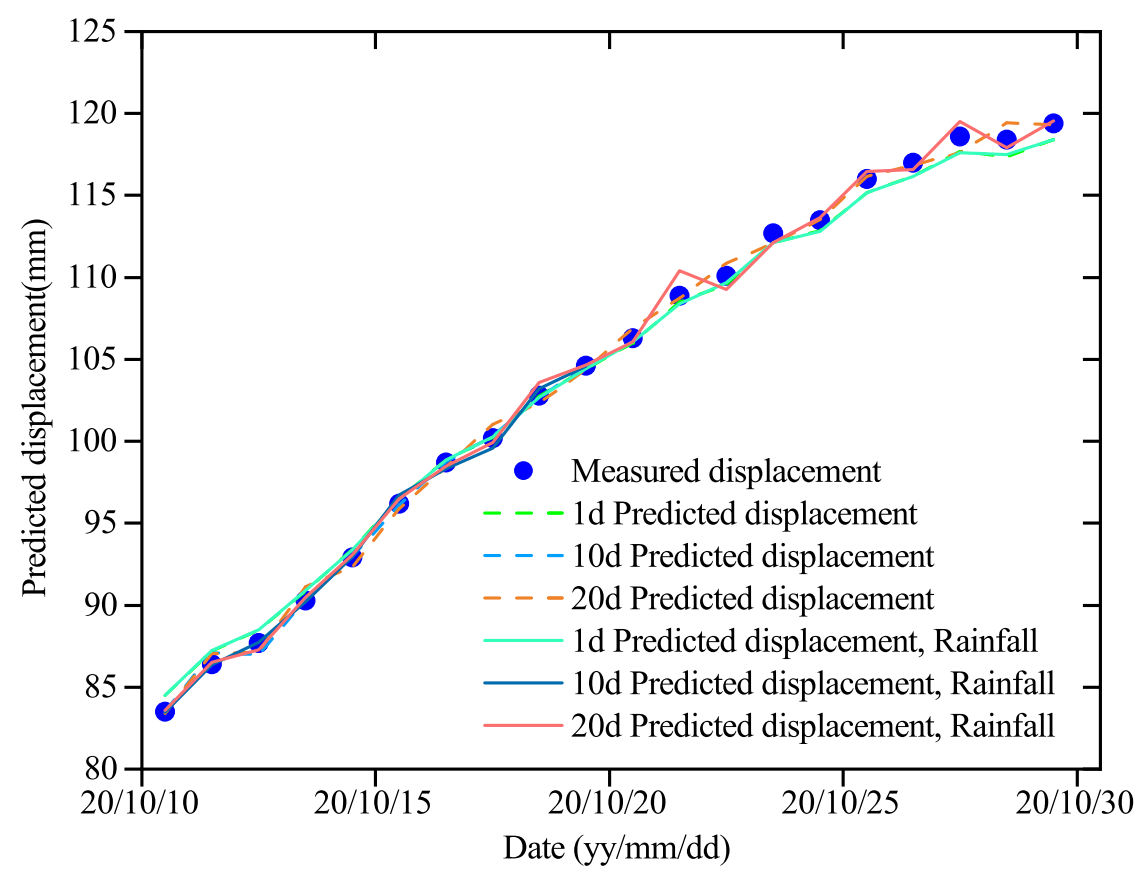

Figure 6. Prediction and comparison of GA-Elman model on test set. 


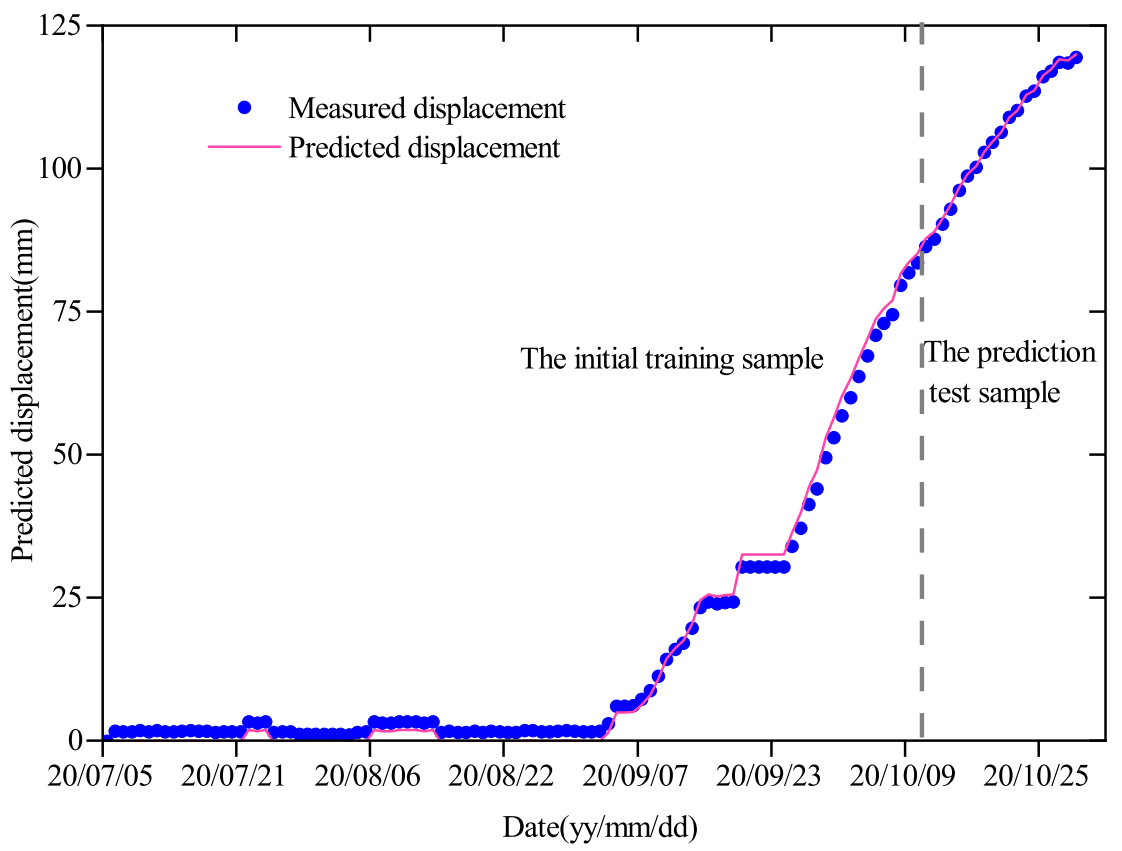

Figure 7. Prediction and comparison of measured displacement and predicted displacement by GA-Elman model.

\subsection{Model Performance Evaluation}

In order to evaluate the predictive performance of the GA-Elman model, the performance indicators in Table 1 are used for evaluation. It can be seen from Table 2 that the RMSE, MAE, MAPE and $\mathrm{R}^{2}$ of the GA-Elman model for 10 consecutive days are $0.314 \mathrm{~mm}$, $0.222 \mathrm{~mm}, 0.243 \%$ and 0.9979 , respectively, which are better than those in other situations. The results show that under the condition of rainfall in flood season, the prediction effect of Jianshanying landslide considering rainfall factors is better than that without considering rainfall factors, which proves that rainfall factors have a certain influence on landslide displacement and shows a certain lag. We can conclude that the accuracy of landslide displacement prediction is relatively high. Considering the landslide displacement prediction is a complex nonlinear problem, the recursive neural network is optimized by GA, and the existing monitoring data are used to train the network. Finally, the nonlinear relationship in the problem of displacement change can be well simulated.

Table 1. Evaluation criteria for prediction performance of machine learning models.

\begin{tabular}{lll}
\hline Item & Formula & Notes \\
\hline RMSE & $R M S E=\sqrt{\frac{1}{n} \sum_{i=1}^{n}\left(\hat{y}_{i}-y_{i}\right)^{2}}$ & $\begin{array}{l}\text { A common measure of the difference between model } \\
\text { predictions } \hat{y}_{i} \text { and actual observations } y_{i}, \text { which can } \\
\text { reflect the accuracy of the prediction model. }\end{array}$ \\
\hline MAE & $M A E=\frac{1}{n} \sum_{i=1}^{n}\left|\hat{y}_{i}-y_{i}\right|$ & $\begin{array}{l}\text { The mean value of the absolute error between the } \\
\text { observed value and the true value, describing the } \\
\text { error between the predicted value and the true value. }\end{array}$ \\
\hline MAPE & $M A P E=\frac{100 \%}{n} \sum_{i=1}^{n}\left|\frac{\hat{y}_{i}-y_{i}}{y_{i}}\right|$ & $\begin{array}{l}\text { A measure of the accuracy of a forecasting method, } \\
\text { usually expressed as a percentage. }\end{array}$ \\
\hline $\mathrm{R}^{2}$ & $R^{2}=1-\frac{\sum_{i=1}^{n}\left(\hat{y}_{i}-y_{i}\right)^{2}}{\sum_{i=1}^{n} \overline{y_{i}}-y_{i}}$ & $\begin{array}{l}\text { A measure mainly used to evaluate the degree of } \\
\text { agreement between the predicted value and the ac- } \\
\text { tual value, its value range is }[0,1] .\end{array}$ \\
\hline
\end{tabular}


Table 2. Performance evaluation of test set prediction using GA-Elman model.

\begin{tabular}{lcccc}
\hline \multicolumn{1}{c}{ Test Set } & RMSE $(\mathbf{m m})$ & MAE $(\mathbf{m m})$ & MAPE $(\%)$ & $\mathbf{R}^{\mathbf{2}}$ \\
\hline 1 day, no rainfall & 0.655 & 0.574 & 0.550 & 0.9967 \\
1 day, Rainfall & 0.658 & 0.579 & 0.556 & 0.9966 \\
10 days, no rainfall & 0.330 & 0.200 & 0.202 & 0.9971 \\
10 days, Rainfall & 0.314 & 0.222 & 0.243 & 0.9979 \\
2 days, no rainfall & 0.567 & 0.472 & 0.446 & 0.9975 \\
20 days, Rainfall & 0.548 & 0.420 & 0.412 & 0.9977 \\
\hline
\end{tabular}

In order to verify the prediction accuracy of the GA-Elman neural network model, comprehensively considering the displacement prediction for 20 consecutive days under rainfall conditions, the Elman neural network prediction method is selected for experimental comparison. Figure 8 shows the comparison between the predicted output of the two models and the measured data. Figure 9 shows the relative error of the predicted percentages of the two models.

The experimental results in Figures 8 and 9 and Table 3 can be seen as follows. (1) Both models can reflect the change of landslide displacement and the error between the prediction results of the model and the measured values is relatively small; (2) The GAElman model overcomes the shortcomings of being easy to fall into the local minimum. The relative percentage error of the GA-Elman model is the smallest and the prediction accuracy is improved compared with the independent Elman network; (3) The accuracy of the prediction results of the two models is greater than 0.995 and the GA-Elman model reaches 0.9977 , indicating that the prediction results of the two models can achieve landslide displacement prediction; the prediction effect of the GA-Elman model is more ideal.

Table 3. Performance evaluation of test set prediction using GA-Elman model.

\begin{tabular}{ccccc}
\hline Model & RMSE (mm) & MAE (mm) & MAPE (\%) & $\mathbf{R}^{\mathbf{2}}$ \\
\hline GA-Elman & 0.548 & 0.420 & 0.412 & 0.9977 \\
Elman & 0.677 & 0.532 & 0.483 & 0.9965 \\
\hline
\end{tabular}

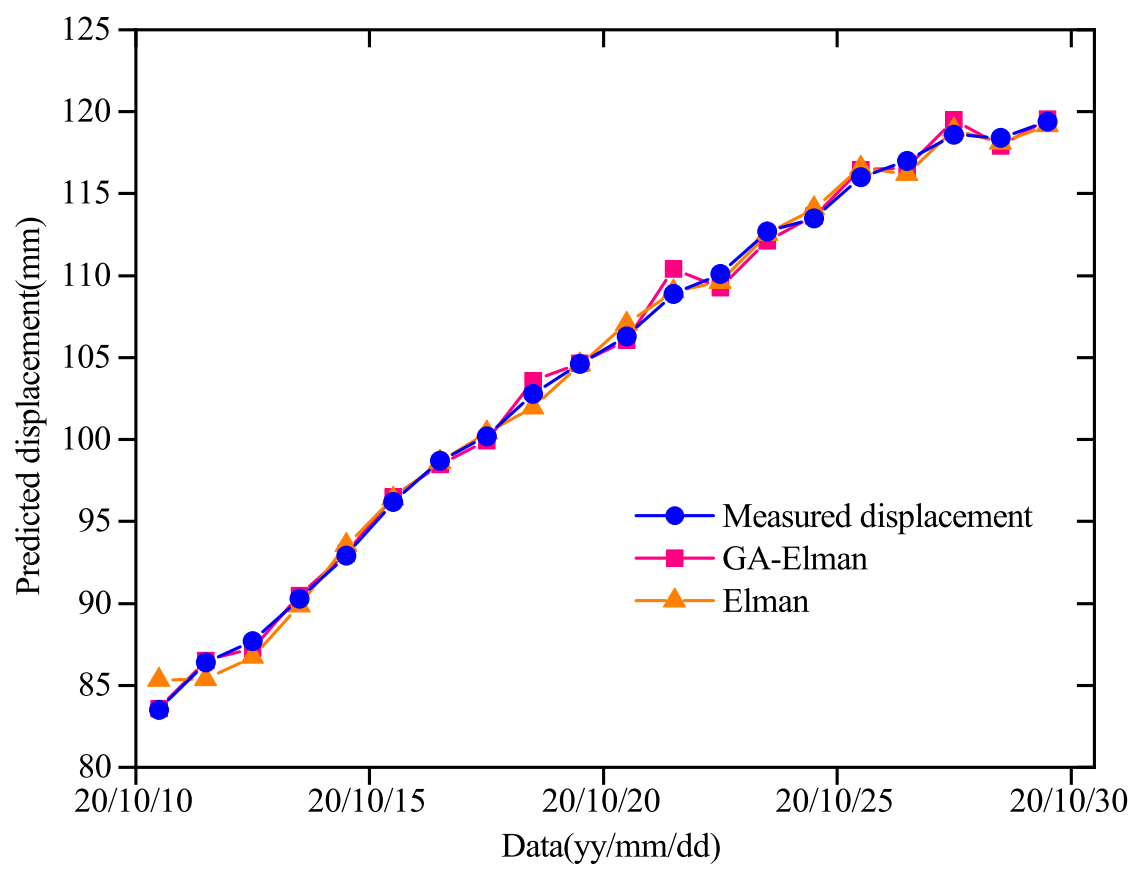

Figure 8. Comparison between prediction output and expectation output. 


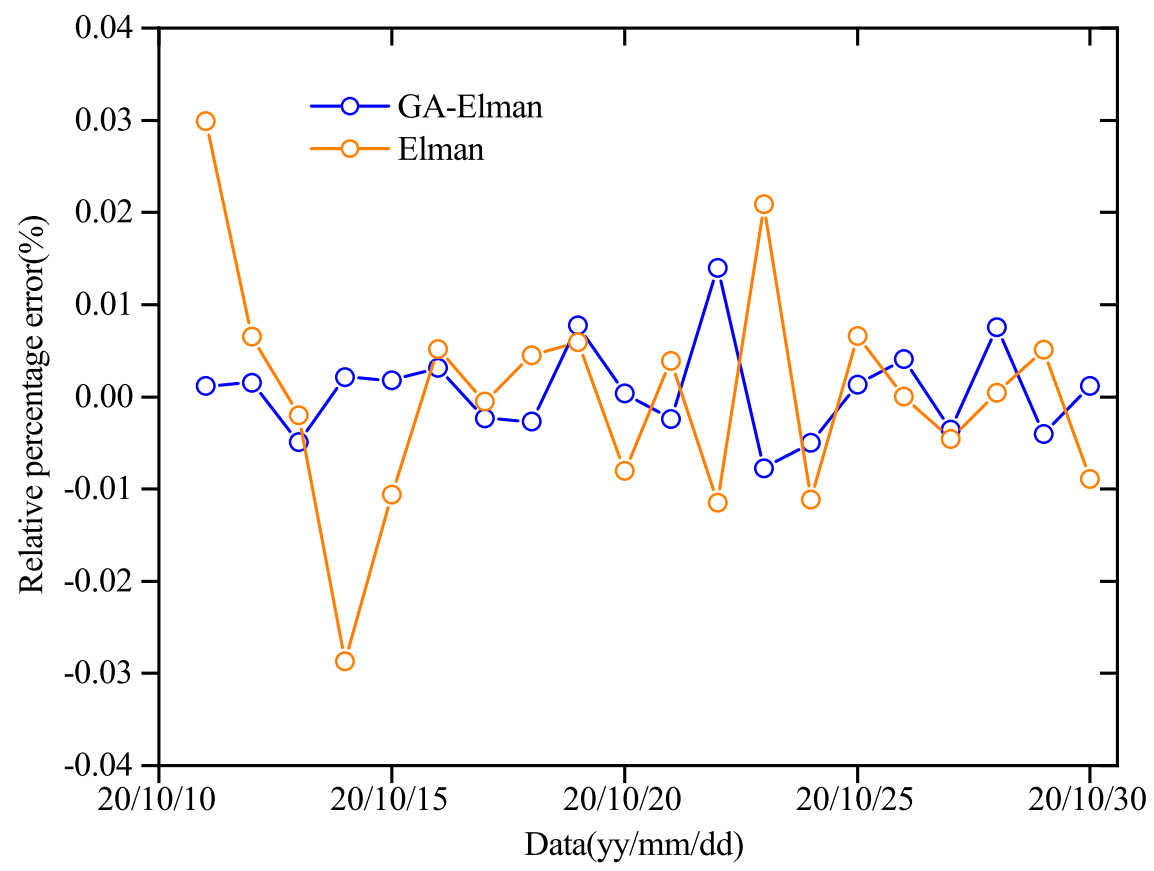

Figure 9. Relative percentage error of prediction with GA-Elman and Elman models.

\section{Discussion and Conclusions}

Based on the displacement monitoring data of Jianshanying landslide in the rainy season in 2020, the model is verified and analyzed by dividing the training set and test set and using the displacement data of the last 20 days. The prediction results show that GA-Elman model can successfully predict landslide displacement and deformation in the case of continuous 1 day, 10 days and 20 days. The preliminary judgment can be made through the model. Under the long-term internal conditions of the landslide, the rainfall factor affects the change of landslide displacement to a certain extent. Considering the six prediction conditions, the prediction effect with rainfall factor is significantly better than that without rainfall factor, so the occurrence of landslide displacement is still affected by rainfall factor. The RMSE, MAE, MAPE and $\mathrm{R}^{2}$ of the test set predicted by GA-Elman for 20 consecutive days under rainfall conditions are $0.548 \mathrm{~mm}, 0.420 \mathrm{~mm}, 0.412 \%$ and 0.9977 , respectively. It can be proved that the model is also suitable for medium and long-term displacement prediction of such landslides. In addition, by comparing the prediction effect of GA-Elman model and Elman neural network model, the results show that the error of GA-Elman model is relatively smaller than that of Elman neural network. The test relative error of GA-Elman model is more accurate than that of Elman neural network. It is proved again that GA-Elman model is superior to the single Elman model in the prediction of landslide displacement. GA-Elman model also has good prediction accuracy, which can achieve accurate prediction of landslide displacement and meet general engineering application requirements. Therefore, according to the displacement and deformation data of the landslide obtained by the GA-Elman prediction model, the deformation rate and deformation acceleration of the landslide can be further calculated. When the predicted value of the landslide deformation reaches the critical early warning index of the landslide, the landslide can be analyzed and judged in time. In this way, the early warning information can be issued in time and the corresponding emergency treatment measures can be taken to effectively reduce casualties and property losses. Overall, because the dynamic deformation process of landslide will be affected by internal and external factors, different models for different influencing factors can effectively improve the prediction accuracy of landslide deformation. Therefore, more other influencing factors (such as climate, groundwater activity, etc.) should be introduced into the model research in future work to further improve the accuracy and precision of landslide displacement prediction. 
Author Contributions: Conceptualization, C.W., Y.Z. and L.B.; methodology, C.W., W.G. and Q.M.; validation, C.W., W.G. and Q.M.; formal analysis, C.W., Y.Z., L.B., W.G. and Meng, Q.; investigation, C.W., W.G. and Q.M.; resources, C.W., W.G. and Q.M.; data curation, C.W., W.G. and Q.M.; writingoriginal draft preparation, C.W., W.G. and Q.M.; writing—review and editing, C.W., W.G. and Q.M.; supervision, Y.Z. and L.B.; project administration, C.W. and Q.M.; funding acquisition, Y.Z. All authors have read and agreed to the published version of the manuscript.

Funding: This research was funded by National Key Research and Development Program of China (No. 2018YFC1504805 and No. 2019YFC1509601), and Geological Survey Projects of China Geological Survey (No. DD20211369 and No. DD20190639).

Institutional Review Board Statement: Not applicable for studies not involving humans or animals. Informed Consent Statement: Not applicable for studies not involving humans.

Acknowledgments: This work was supported by National Key Research and Development Program of China (No. 2018YFC1504805 and No. 2019YFC1509601), Geological Survey Projects of China Geological Survey (No. DD20211369 and No. DD20190639).

Conflicts of Interest: The authors declare no conflict of interest. The funders had no role in the design of the study; in the collection, analyses, or interpretation of data; in the writing of the manuscript, or in the decision to publish the results.

\section{References}

1. Segoni, S.; Piciullo, L.; Gariano, S.L. Preface: Landslide early warning systems: Monitoring systems, rainfall thresholds, warning models, performance evaluation and risk perception. Nat. Hazards Earth Syst. Sci. 2018, 18, 3179-3186. [CrossRef]

2. Zhu, X.; Xu, Q.; Tang, M.; Nie, W.; Ma, S.; Xu, Z. Comparison of two optimized machine learning models for predicting displacement of rainfall-induced landslide: A case study in Sichuan Province, China. Eng. Geol. 2017, 218, 213-222. [CrossRef]

3. Miao, S.; Hao, X.; Guo, X.; Wang, Z.; Liang, M. Displacement and landslide forecast based on an improved version of Saito's method together with the Verhulst-Grey model. Arab. J. Geosci. 2017, 10, 1-10. [CrossRef]

4. Du, H.; Song, D.; Chen, Z.; Shu, H.; Guo, Z. Prediction model oriented for landslide displacement with step-like curve by applying ensemble empirical mode decomposition and the PSO-ELM method. J. Clean. Prod. 2020, 270, 1-17. [CrossRef]

5. Zhou, C.; Yin, K.; Cao, Y.; Intrieri, E.; Ahmed, B.; Catani, F. Displacement prediction of step-like landslide by applying a novel kernel extreme learning machine method. Landslides 2018, 15, 2211-2225. [CrossRef]

6. Alessandro, C.; Marco, M. Use of ROC curves for early warning of landslide displacement rates in response to precipitation (Piagneto landslide, Northern Apennines, Italy). Landslides 2016, 14, 1241-1252.

7. Deng, J. Grey Prediction Decision, 1st ed.; Huazhong University Technology Press: Wuhan, China, 1986; pp. 10-43.

8. Yin, K.; Yan, T. Landslide prediction and relevant models. Chin. J. Rock Mech. Eng. 1996, 15, 1-8.

9. Qin, S. Nonlinear evolutionary mechanisms and physical prediction of instability of planar-slip slope. Chin. J. Geotech. Eng. 2005, $27,1241-1248$.

10. Sangeeth, K.; Venkat, R.P.; Vinodini, R.M. Design and Validation of Wireless Communication Architecture for Long Term Monitoring of Landslides. Adv. Cult. Living Landslides 2005, 51-60.

11. Du, J.; Yin, K.; Lacasse, S. Displacement prediction in colluvial landslides, Three Gorges Reservoir, China. Landslides 2012, 10, 203-218. [CrossRef]

12. Yao, W.; Zeng, Z.G.; Lian, C.; Tang, H. Training enhanced reservoir computing predictor for landslide displacement. Eng. Geol. 2015, 188, 101-109. [CrossRef]

13. Cai, Z.; Xu, W.; Meng, Y.; Shi, C.; Wang, R. Prediction of landslide displacement based on GA-LSSVM with multiple factors. Bull. Eng. Geol. Environ. 2015, 75, 637-646. [CrossRef]

14. Huang, F.; Yin, K.; Zhang, G.; Gui, L.; Yang, B.; Liu, L. Landslide displacement prediction using discrete wavelet transform and extreme learning machine based on chaos theory. Environ. Earth Sci. 2016, 75, 1376. [CrossRef]

15. Huang, F.; Huang, J.; Jiang, S.; Zhou, C. Landslide displacement prediction based on multivariate chaotic model and extreme learning machine. Eng. Geol. 2017, 218, 173-186. [CrossRef]

16. Huang, D.; Gu, D.M.; Song, Y.X.; Cen, D.F.; Zeng, B. Towards a complete understanding of the triggering mechanism of a large reactivated landslide in the Three Gorges Reservoir. Eng. Geol. 2018, 238, 36-51.

17. Huang, F.; Luo, X.; Liu, W. Stability Analysis of Hydrodynamic Pressure Landslides with Different Permeability Coefficients Affected by Reservoir Water Level Fluctuations and Rainstorms. Water 2017, 9, 450. [CrossRef]

18. Ma, J.; Tang, H.; Liu, X.; Wen, T.; Zhang, J.; Tan, Q.; Fan, Z. Probabilistic forecasting of landslide displacement accounting for epistemic uncertainty: A case study in the Three Gorges Reservoir area, China. Landslides 2018, 15, 1145-1153. [CrossRef]

19. Ma, J.; Tang, H.; Liu, X.; Hu, X.; Sun, M.; Song, Y. Establishment of a deformation forecasting model for a step-like landslide based on decision tree C5.0 and two-step cluster algorithms: A case study in the Three Gorges Reservoir area, China. Landslides 2017, 14, 1275-1281. [CrossRef] 
20. Huang, G.; Zhu, Q.; Siew, C. Extreme learning machine: Theory and applications. Neurocomputing 2006, 70, 489-501. [CrossRef]

21. Lian, C.; Zeng, Z.; Yao, W.; Tang, H. Extreme learning machine for the displacement prediction of landslide under rainfall and reservoir level. Stoch. Environ. Res. Risk Assess. 2014, 28, 1957-1972. [CrossRef]

22. Lian, C.; Zeng, Z.; Yao, W.; Tang, H. Multiple neural networks switched prediction for landslide displacement. Eng. Geol. 2015, 186, 91-99. [CrossRef]

23. Lian, C.; Zeng, Z.; Yao, W.; Tang, H. Ensemble of extreme learning machine for landslide displacement prediction based on time series analysis. Neural Comput. Appl. 2013, 24, 99-107. [CrossRef]

24. Elman, J.L. Finding Structure in Time. Cogn. Sci. 1990, 14, 179-211. [CrossRef]

25. Bai, X.; Liang, D.; Ma, Y. Applied Research in the Deformation Monitoring Based on Elman Neural Network Method. Geomat. Spat. Inf. Technol. 2012, 35, 156-158.

26. Chen, H.; Zeng, Z.; Tang, H. Landslide Deformation Prediction Based on Recurrent Neural Network. Neural Process. Lett. 2013, 41, 169-178. [CrossRef]

27. Li, X.; Ye, J.; Li, G.; Li, J. Elman neural network dynamic prediction based on landslide monitoring data. Coal Geol. Explor. 2018, $35,113-119+126$.

28. Li, S.; Han, Y.; Yang, H.; Li, Y. Research on LMD-Elman-based time-series rolling prediction of slope deformation in open-pit mine. China Saf. Sci. J. 2015, 25, 22-28.

29. Yao, Z.; Xu, J.P.; Kong, J.L.; Liu, S.B. Prediction of River Water Level by GA-Elman Model. J. Yangtze River Sci. Res. Inst. 2018, 35, 34-37.

30. Li, X.Z.; Kong, J.M. Application of GA-SVMmethodwith parameter optimization for landslide development prediction. Nat. Hazards Earth Syst. 2014, 14, 525-533. [CrossRef]

31. Zhang, Q.; Zhu, X. Short-time traffic flow forecasting method based on GA-Elman neural network. J. Lanzhou Univ. Technol. 2013, 39, 94-98.

32. Zhu, M.; Lu, Q.; Ding, Y.M. Effectiveness evaluation for underwater unmanned swarmcombat based on GA-Elman neural network. Fire Control. Command. Control 2020, 45, 115-119.

33. Behzad, M.; Hossein, I.; Hossein, M. Shear wave velocity prediction using Elman artificial neural network. Carbonates Evaporites 2017, 34, 1281-1291.

34. Shiluo, X.; Ruiqing, N. Displacement prediction of Baijiabao landslide based on empirical mode decomposition and long short-term memory neural network in Three Gorges area, China. Comput. Geosci. 2018, 111, 87-96.

35. Grefenstette, J. Optimization of Control Parameters for Genetic Algorithms. IEEE Trans. Syst. Man Cybern. 1986, 16, 122-128. [CrossRef] 